\title{
Nuestra experiencia en el priapismo de alto flujo por fístula arterio-lacunar uni y bilateral
}

\author{
Rodriguez Tolrá J, Cuadrado Campaña JM, Ropero Valverde J, Ruiz Salas V, Franco Miranda E.
}

Unidad de Andrología. Servicio de Urología. Hospital Univ. de Bellvitge. L’Hospitalet. Barcelona.

Actas Urol Esp. 2007;31(2):113-119

\section{RESUMEN}

\section{NUESTRA EXPERIENCIA EN EL PRIAPISMO DE ALTO FLUJO POR FÍSTULA ARTERIO-LACUNAR UNI Y BILATERAL}

Objetivo: Presentar nuestra experiencia en el priapismo de alto flujo tanto uni como bilateral, resaltando los buenos resultados obtenidos con la embolización supraselectiva.

Material y método: Presentamos 5 casos de priapismo de alto flujo secundarios a traumatismo perineal, con una edad media de 31 años (24-43 a). El tiempo medio de consulta desde el momento del traumatismo fue de 18,6días (1-60 d). El diagnóstico se confirmo mediante gasometría de los cuerpos cavernosos, eco-doppler peneano (2 casos) y arteriografia selectiva de la pudenda. En todos los casos el tratamiento fue la embolización supraselectiva con esponja de gelatina. En los dos casos bilaterales la embolización se realizo en el mismo acto.

Resultados: A corto plazo fueron la recuperación de la flacidez y a largo plazo (3-4 meses) la recuperación de la erección sin secuelas fibróticas de los cuerpos cavernosos en todos los casos.

Conclusiones: La embolización de la arteria lacerada, y en caso de que sea bilateral en un solo tiempo, reporta excelentes resultados.

Palablas clave: Priapismo de alto flujo. Fístula arterio-lacunar. Embolización.

\section{ABSTRACT}

\section{OUR EXPERIENCE IN HIGH-FLOW PRIAPISM DUE TO UNI-OR BILATERAL ARTERIO-LACUNAR FISTULA}

Objective: To present our experience in both uni-and bilateral priapism, highlighting good results obtained with supraselective embolisation.

Material and methods: We present 5 cases of high-flow priapism secondary to perineal trauma, with a mean age of 31 years (24-43 years). The mean time to presentation from the moment of the trauma was 18,6 days (1-60 days). Diagnosis was confirmed through gasometry of the corpora cavernosa, penile doppler ultrasound ( 2 cases) and selective arteriography of the pudendal artery. In all cases treatment was by supralective embolisation with gelatin sponge. In the two bilateral cases, embolisation was performed in the same act.

Results: In the short term was a recovery of flaccidity and in the long term (3-4 months) a recovery of erection with no fibrotic sequelae of the corpora cavernosa.

Conclusions: Embolisation of the lacerated artery, in a single procedure in cases of bilateral lacerations, provides excellent results. 
$\mathrm{C}$ lásicamente el priapismo se conoce como una erección peneana persistente y dolorosa, limitada a los cuerpos cavernosos y sin estar asociada al deseo sexual. Hay dos clases de priapismo. El mas conocido es el de bajo flujo o venoso, llamado así porqué hay un compromiso del retorno venoso que ocasiona hipoxia y una acidosis, que en último termino serán los responsables de la muerte celular y la posterior fibrosis. El otro tipo de priapismo es el denominado de alto flujo (termino que introdujo Hauri en $1983^{1}$.y que tiene su causa mas frecuente en un traumatismo perineal o peneano con la consiguiente laceración de la arteria cavernosa o de una se sus ramas y la aparición de una fístula arterio-lacunar ${ }^{2,3}$, no debemos olvidar que también puede producirse por la laceración de la pared arterial durante una inyección intracavernosa ${ }^{2,3}$, durante una cirugía de revascularización peneana o bien en el tratamiento quirúrgico del priapismo veno-oclusivo mediante shunts cavernoso-esponjosos, que laceran la arteria cavernosa y convierten el priapismo venooclusivo en arterial ${ }^{4}$. La fístula arterio-lacunar produce un flujo de sangre turbulento sobre las células endoteliales que tapizan los espacios lacunares, el consiguiente aumento de la oxigenación por el alto flujo favorece la sintesis y liberación de óxido nítrico por el endotelio, causando de éste modo la vasodilatación del cuerpo cavernoso. No obstante, ésta distensión del espacio lacunar produciría, al no haber estímulo neurológico, una compresión parcial de la albugínea sobre el sistema venoso, causando una reducción incompleta del retorno venoso por falta de relajación neurogénica del músculo trabecular, lo cual permitiría que hubiese un alto flujo de entrada, pero con salida de los cuerpos cavernosos ${ }^{2}$. Por tanto el pronóstico del priapismo de alto flujo es bueno, ya que no hay isquemia ni sufrimiento tisular, que en definitiva son los dos factores que complican el pronóstico en el priapismo veno-oclusivo.

Teniendo en cuenta lo expuesto, el objetivo del trabajo, sería demostrar que con un tratamiento adecuado, los pacientes con priapismo de alto flujo mantienen su función eréctil.

\section{MATERIAL Y MÉTODOS}

Se trata de un estudio retrospectivo realizado entre enero de 1991 y diciembre del 2002 en el que se diagnosticaron 5 casos de priapismo de alto flujo, 2 bilaterales (Pacientes J.M. Fig. 1, 2 y E.D.) y 3 unilaterales (Pacientes P.A. Fig. 3, M.H.
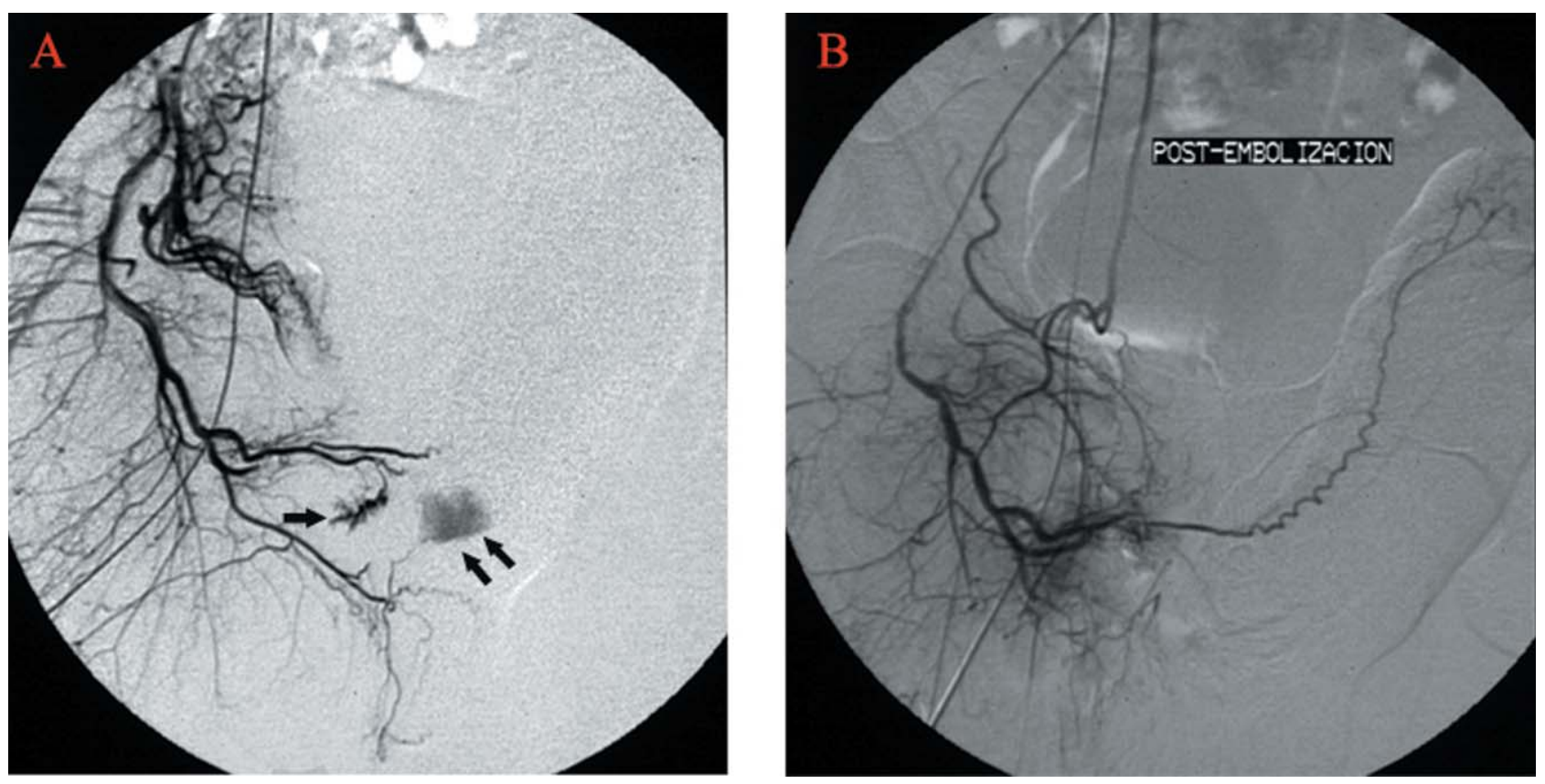

FIGURA 1. Paciente J.M. Arteriografia selectiva de la pudenda derecha. A. Fuga de contraste de la art. cavernosa (1 flecha), fuga de contraste de la art. bulbo-uretral (2 flechas).

B. Desaparición de las fugas de contraste después del tratamiento. 

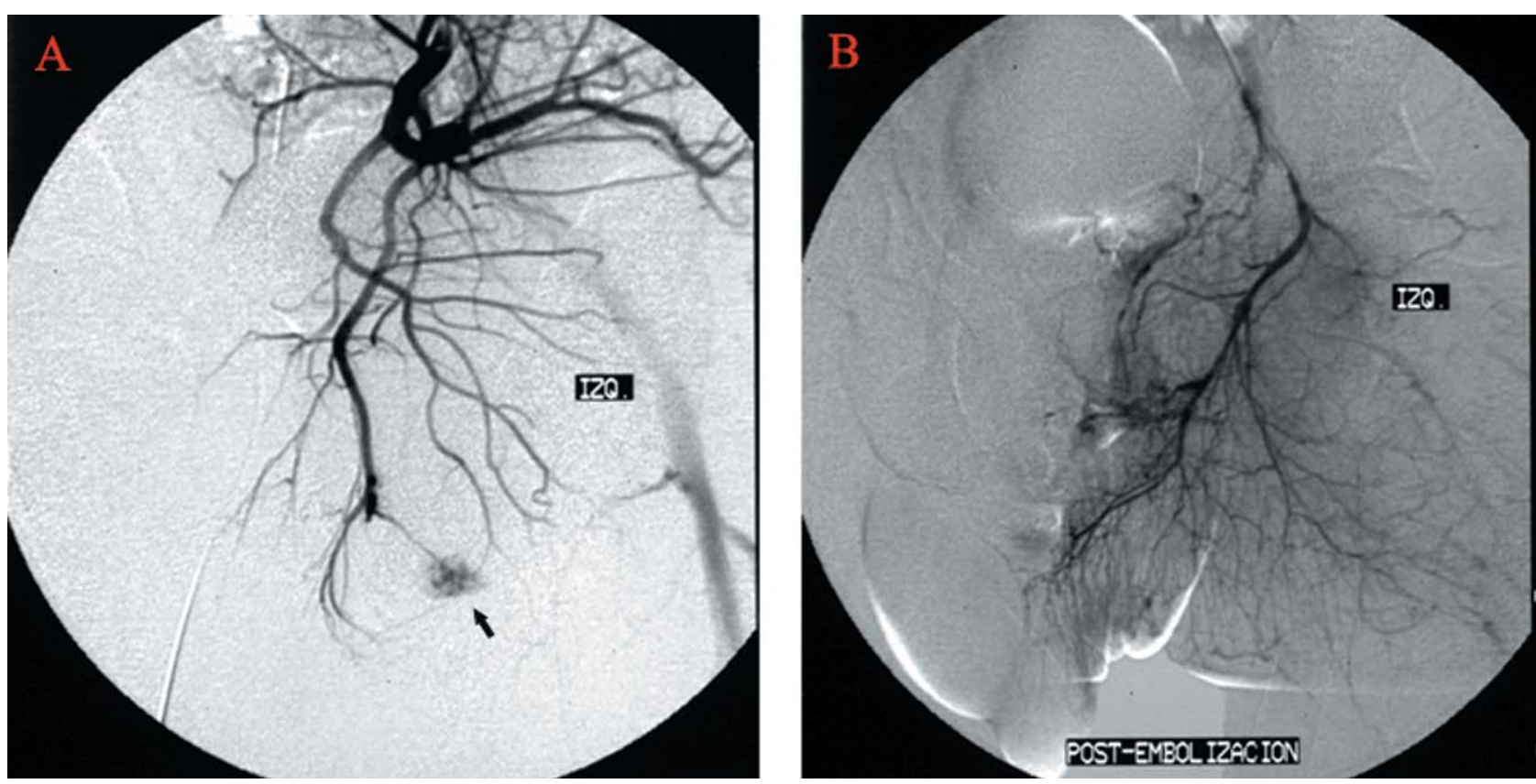

FIGURA 2. Paciente J.M. Arteriografía de la pudenda izquierda. A. Fuga de contraste de la arteria cavernosa. B. Desaparición de la fuga de contraste después del tratamiento.
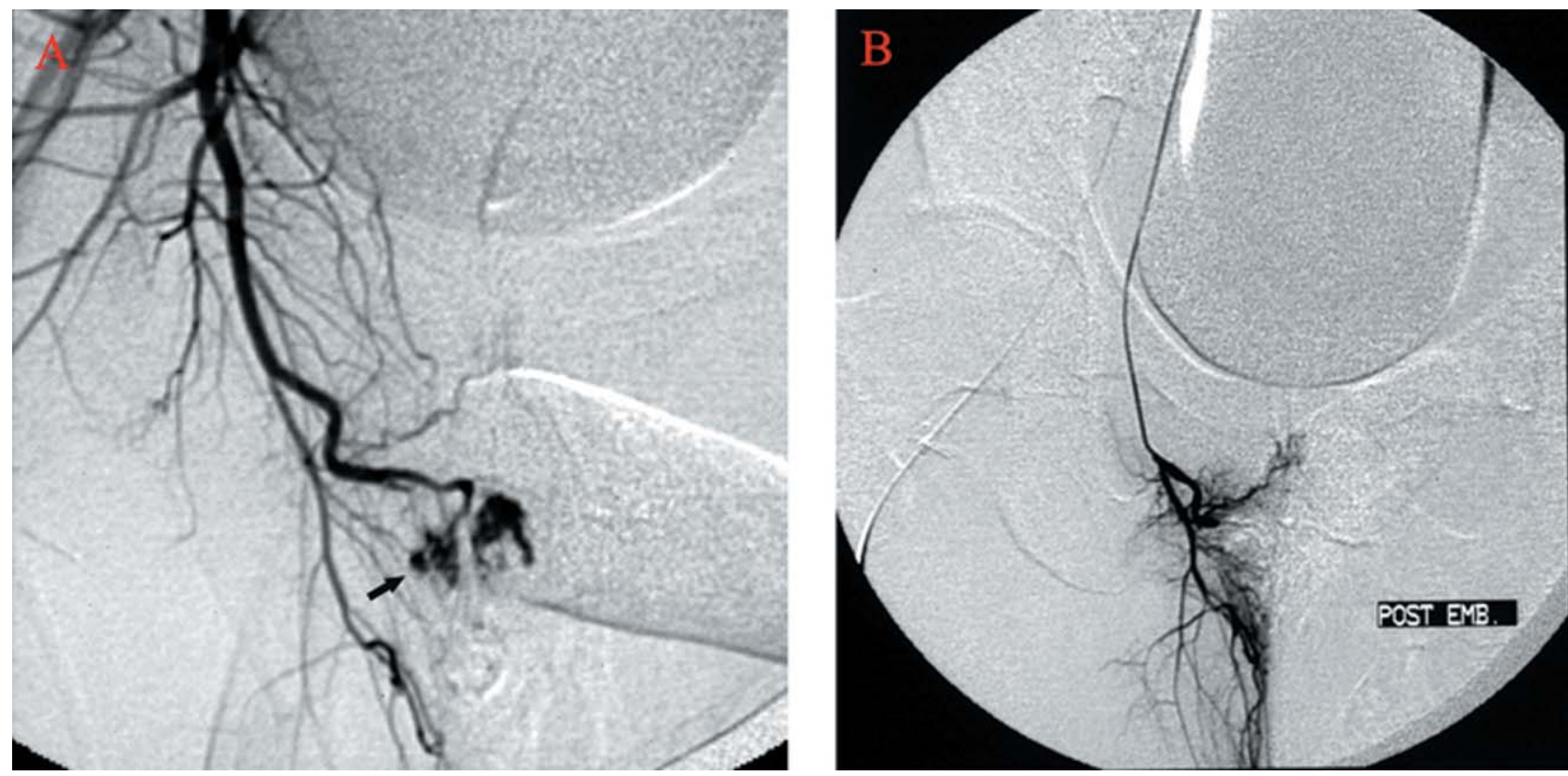

FIGURA 3. Paciente P.A. Arteriografía con estravasación de contraste al espacio lacunar del cuerpo cavernoso B. Desaparición de la fuga de contraste después de la embolización.

Fig. 4 y J.B.) La etiología en todos ellos fue debida a un traumatismo perineal (en dos casos por accidente de circulación). La edad media fue de 31 años (24-43 a). En todos los casos se podía apreciar una tumescencia peneana con una rigidez que no era total y ausencia de dolor. El tiem- po transcurrido desde el traumatismo hasta la consulta oscilo entre 1-60 días (media: 18,6 días). El diagnóstico aparte de valorar los datos clínicos, se confirmó mediante la gasometría de la sangre de los cuerpos cavernosos, que en todos los casos dio valores típicos de sangre arterial. 

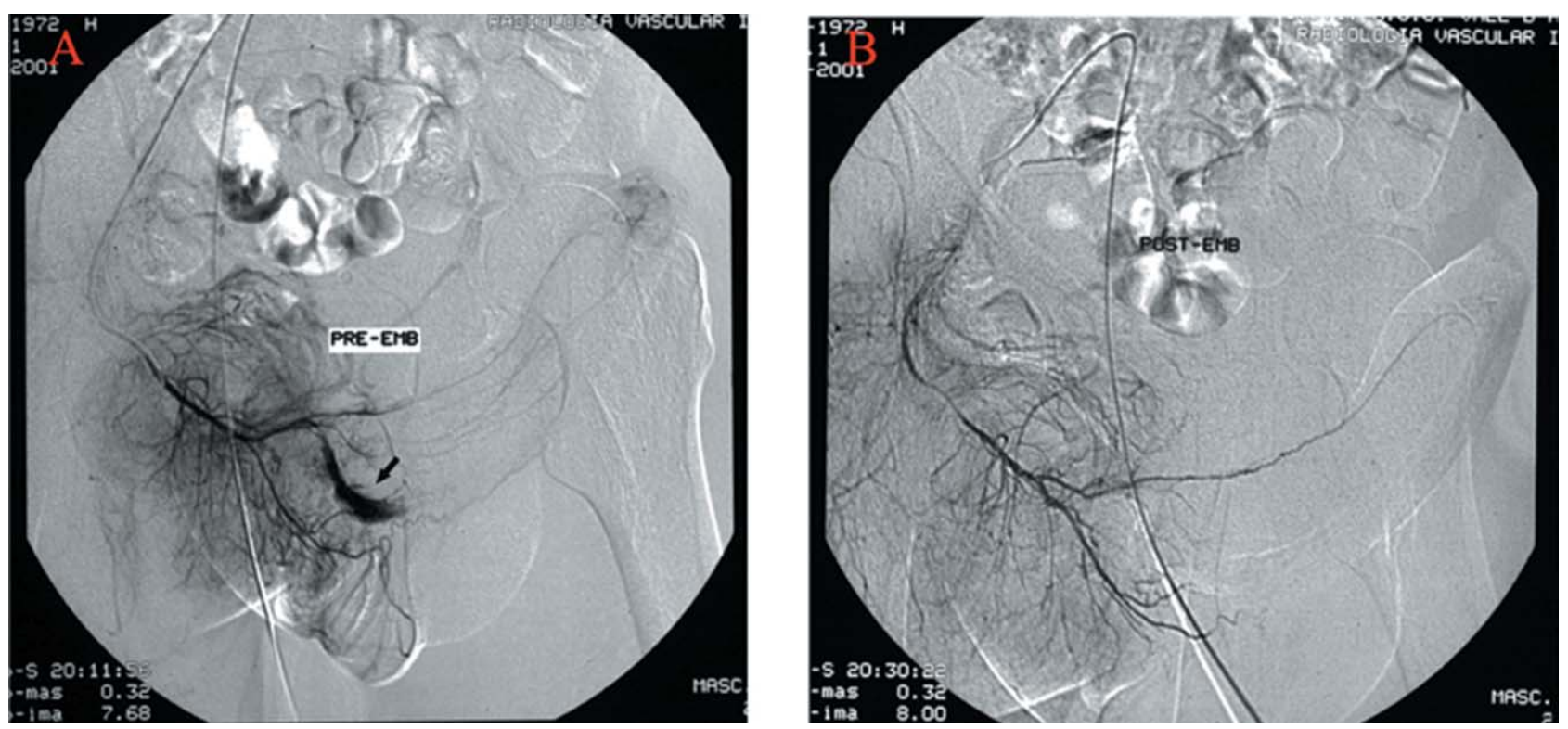

FIGURA 4. Paciente M.H. A. Arteriografia de la prueba izquierda con fístula arterio-cavernosa. B. Arteriografia de la pudenda izquierda. La fistula arterio-cavernosa desaparece después de la embolización.

En los dos últimos casos el diagnóstico se completó con eco-doppler peneano en el que se apreció un flujo turbulento los cuerpos cavernosos afectados y que nos orientó de la uni o bilateralidad del proceso. En todos los casos se realizó arteriografia selectiva de la pudenda que fue a la vez diagnóstica y terapéutica, ya que se embolizó la arteria lacerada con material reabsorbible (esponja de gelatina). En los dos casos bilaterales, la embolización se realizó en el mismo acto. Entre el diagnóstico y el tratamiento el tiempo trascurrido osciló entre 2 y 4 días (media 2,8 días).

\section{RESULTADOS}

Después de la embolización se produjo en todos los casos una desaparición rápida del priapismo a las pocas horas. La recuperación completa de la erección, también en todos los casos, se produjo a los 3-4 meses de la embolización. El hecho de la recuperación completa de la erección sin secuelas, se debe a que el priapismo de alto flujo no deja secuelas fibróticas en los cuerpos cavernosos.

\section{DISCUSIÓN}

El priapismo de alto flujo es un proceso patológico poco frecuente. El origen suele ser un traumatismo perineal que ocasiona una laceración de la arteria cavernosa y la formación de una fístula arterio-lacunar, en la cual la sangre pasa directamente al espacio lacunar puenteando las arterias helicinas. Por tanto no se trata de una fístula arterio-venosa tradicional en que la sangre pasa directamente del sistema arterial al venoso corto-circuitando la red capilar ${ }^{3}$. La sospecha de este tipo de priapismo vendrá dada por la anamnesis y la exploración física (erección no dolorosa e incompleta). La confirmación diagnóstica se hará mediante la gasometría de la sangre de los cuerpos cavernosos, que dará parámetros de sangre arterial. Llegado este punto diremos que en la actualidad se hace necesario el uso del ecodoppler peneano, que pondrá de manifiesto la existencia de un flujo turbulento ocasionado por la fístula arterio lacunar ${ }^{5-8}$. Quizás lo mas importante de él será el orientarnos hacia la lateralidad del proceso e indicarnos la bilateralidad cuando ésta exista. Nosotros lo utilizamos siempre en los últimos años y nos sirvió para diagnosticar uno de los dos casos bilaterales. Volkmer ${ }^{7}$ lo utiliza combinado con la arteriografía para disminuir el tiempo de exposición radiológico y la cantidad de contraste yodado. También se ha utilizado después de la arteriografía para confirmar el buen resultado de la embolización ${ }^{6}$. El último paso diagnóstico será la arteriografia selectiva de la pudenda, que objetivará la extravasación de con- 
traste al cuerpo cavernoso desde el sistema arterial. Esta prueba nos permitirá también demostrar la bilateralidad o no del proceso, pero solo debemos realizarla como paso previo a la embolización y no como método diagnóstico ${ }^{9}$.Últimamente algunos autores han utilizado para el diagnóstico, la CT sin contraste ${ }^{10} \mathrm{o}$ la angio-resonan$\mathrm{cia}^{11}$. De entre los varios tratamientos propuestos, algunos autores aconsejan, una vez realizado el diagnóstico, la conducta expectante, y lo hacen porqué sus casos se solucionaron con esta pauta $^{12,13}$. En un caso de fistula bilateral la conducta expectante permitió que se solucionara espontáneamente un lado $^{14}$. Si bien esta conducta puede ser efectiva en algunos casos, lo mas frecuente es que la erección se mantenga durante meses o años, este hecho junto a la ansiedad e intolerancia que suele ocasionar la idea de una erección permanente, desaconsejan optar por esta pauta como de elección ${ }^{3}$. Hay autores que abogan por la compresión externa prolongada del periné durante varias horas, Hatzichristou ${ }^{15}$ de 7 casos consigue solucionar 1 por este método, pero no parece que vaya a ser útil en muchos casos.

El uso de algunos fármacos alfa-adrenérgicos, tampoco parece muy adecuado. Moscovici ${ }^{16}$ lo ha usado sin buenos resultados, Witt ${ }^{2}$ consiguió detumescencia transitoria, y solo publican buenos resultados Gudinchet ${ }^{17}$ utilizando epinefrina diluida y Mizutani ${ }^{18}$ con metaraminol. Nosotros pensamos que en el priapismo de alto flujo el uso de estas sustancias, que no quedan atrapadas en el pene, pueden ocasionar complicaciones sistémicas secundarias a la difusión general del fármaco, por lo que no aconsejamos su uso. El tratamiento de elección en la actualidad será la embolización selectiva de la rama arterial aferente a la fístula lacunar. La discusión vendría en primer lugar por el tipo de material a utilizar para la embolización, y en segundo lugar, se plantearía la conveniencia de la embolización en uno o dos tiempos en aquellos casos en que la fístula fuera bilateral. Los materiales utilizados para la embolización los podríamos dividir entre: reabsorbibles e irreabsorbibles. Dentro de los primeros se ha utilizado el coágulo autólogo $0^{2,3,19-25}$ y la esponja de gelatina ${ }^{22,26-34}$. Los materiales reabsorbibles producen una interrupción transitoria del flujo arterial a través del vaso lacerado, con revascularización arterial posterior y recuperación completa de la capacidad eréctil. Nosotros hemos utilizado en todos los casos esponja de gelatina. Entre los materiales no-reabsorbibles se ha utilizado el bucrilato ${ }^{35}$, pero lo mas frecuente es utilizar espirales metálicos (coils de platino ${ }^{31,32,36-40}$. Ha habido un autor que ha utilizado una emulsión de oclusión irreabsorbible ${ }^{41}$. Cuando se utiliza el material irreabsorbible se pretende una embolización definitiva y parece ser que los resultados en cuanto a recuperación de la capacidad eréctil son igualmente buenos. En los casos de fistula bilateral, el tratamiento a aplicar es el mismo, no obstante hay muy pocos descritos en la literatura, ya que contabilizando nuestros dos casos, sumamos solamente 14, 11,12,30,31,36,37,40-44. El dilema estaría en si la embolización la hemos de realizar en uno o dos tiempos. Inicialmente se abogaba para que se realizara en dos tiempos, con material reabsorbible y con una diferencia de varias semanas, esperando a que la primera arteria embolizada se recanalizase, sin embargo, al revisar los casos publicados, solo uno se atiene a estas normas terapéuticas recomendadas, utilizando por tanto coagulo autólogo como material embolizante y con un intervalo de tiempo entre ambos lados de 15 días $^{43}$. En el resto de casos encontramos diversidad de posturas, en un caso se opto por una actitud conservadora ${ }^{11}$, en otro caso la actitud conservadora funcionó en un lado, convirtiéndose en un proceso unilateral que se embolizó ${ }^{12}$. La embolización con material irreabsorbible se utilizó por 4 autores ${ }^{7,31,36,37,40}$ en un total de 5 casos. En los 6 pacientes restantes se empleo esponja de gelatina en embolización bilateral y en un sólo procedimiento en 5 de ellos, (nuestros dos casos y ${ }^{30,42,44}$ ) y sin respetar el periodo de tiempo necesario para la reabsorción en el otro caso ${ }^{31}$. Por tanto si tenemos encuentra tanto los resultados publicados como nuestra propia experiencia, sobre la recuperación de la función eréctil, podemos decir que la embolización en un solo tiempo en los casos de fistula bilateral es segura. Es importante comentar también que la embolización no es efectiva en el 100 $\%$ de los casos y que en algunos hay que realizar un segundo intento ${ }^{7,45,46}$ incluso hay un autor que presenta un caso con tres intentos de embo- 
lización que no resultaron efectivos y que necesitó al final una ligadura ${ }^{46}$. La ligadura de la arteria cavernosa o pudenda interna también se ha descrito como terapéutica efectiva ${ }^{3,15,38,47}$, no obstante esta técnica tiene dos importantes desventajas: el posible daño del tejido cavernoso durante la exploración y la no repermeabilización arterial, por tanto creemos que solo debe aplicarse cuando falla la embolización, entre otros aspectos porque desconocemos sus efectos sobre la erección a largo plazo.

\section{CONCLUSIONES}

El priapismo de alto flujo es un proceso que una vez diagnosticado no precisa de una actuación terapéutica urgente. Si se decide una actitud conservadora, no debería utilizarse la arteriografia solo como método diagnóstico, y realizar ésta solamente con fines terapéuticos. Creemos que la embolización de la arteria lacerada es el tratamiento de elección y que en casos de fístula bilateral realizarla en un solo tiempo es seguro. Nosotros abogamos para que se realice inicialmente con material reabsorbible, dejando el material irreabsorbible y el abordaje quirúrgico para cuando falle la embolización con el primero. También puede sacarse como conclusión que al no existir isquemia ni sufrimiento tisular, no aparecerán fenómenos de fibrosis en el tejido cavernoso, y por tanto la erección se recuperara completamente en un periodo de 3 a 4 meses.

\section{REFERENCIAS}

1. Hauri D, Spycher M., Brulhmann W. Erection and priapism: a new physiopathological concept. Urol Int. 1983;38(3):138-145.

2. Witt MA, Goldstein I., Saenz de Tejada I., Greenfield A, Krane RJ. Traumatic laceration of intracorporal arteries: The pathophiyology of non ischemic, high-flow priapism. J Urol. 1990;143(1):129-132.

3. Bastuba MD, Saenz de Tejada I, Dinlenc CZ, Sarazen A, Krane RJ, Goldstein I. Arterial priapism: Diagnosis, treatment and long-term followur. J Urol. 1994;151(5):1231-1237.

4. Negrin Diaz A, Diaz Romero F, Fernandez Ramos J, Gutierrez Hernandez P, Arteaga Serrano F, Banares Baudet F. Priapismo de éstasis, secundario a PGE1: Transformación en priapismo de alto flujo. Actas Urol. Esp. 1996;20(3): 289-291.

5. Gudinchet D, Fournier D, Jichlinski P, Meyrat B. Traumatic priapism in child: Evaluation with color flow doppler sonography. J Urol. 1992;148:380(2 Pt 1)-381.

6. Bertolotto M, Quaia E, Mucelli FP, Ciampalini S, Forgacs B, Gattuccio I. Color doppler imaging of posttraumatic priapism before and after selective embolization. Radiografics 2003;23(2):495-503.
7. Wolkmer BG, Nesslauer T, Kuefer R. Kraemer S, Goerich J, Gottfried HW. High-flow priapism: A combined interventional approach with angiography and colour doppler source. Ultrasound in medicine \& Biology 2002;28(2): 165-169.

8. Eracleous E, Kondou M, Aristidou K, Eliades S, Pantziaris $\mathrm{M}$, Posporis T, et al. Use of doppler ultrasound and 3dimensional contrast-enhanced MR angiography in the diagnosis and follow-up of post-traumatic high-flow priapism in a child. Pediatric Radiology 2000;30(4):265-267.

9. Caballero Gómez M, Cuervo Pinna C, Abengózan GarciaMoreno A, Fernandez Alarcon L, Villa Minguez D, Murillo Mirat J, Dominguez Bravo C. Priapismo de alto flujo: Revisión y aportación de un caso. Actualidad Andrológica 1997(3): 101.

10. Suzuki K, Nishizawa S, Muraishi O, Fujita A, Hyodoh H, Tokue A. Posttraumatic high-flow priapism: demostrable findings of penile enhanced computed tomography. Inter Jour of Urology 2001;8(11):648-651.

11. Eracleous E, Kondou M, Aristidou K, Eliade S, Pantziaris $\mathrm{M}$, Posporis T, et al. Use of doppler ultrasound and 3dimensional contrast-enhanced MR angiography in the diagnosis and follow-up of post-traumatic high-flow priapism in a child. Pediatric Radiology 2000;30(4):265267.

12. Arango O, Castro R, Domínguez J, Gelabert A. Complete resolution of post traumatic high-flow priapism with conservative treatment. Int J Impot Res. 1999;11(2):115-117.

13. Ficarra V, Beltrami P, Sarti A, Rubilotta E, Righetti R, Malossini G. High-flow priapisme due to a bilateral arteriosinusoidal fistule: An inusual conservative treatment. Scand J Urol Nephrol. 2001;35(5):418-419.

14. Goktas S, Tahmaz L, Atac K, Erduran D, Ogur E. High-flow priapism due to bilateral arteriosinusoidal fistulae. $\mathrm{Br} \mathrm{J}$ Urol. 1996;77(1):165-166.

15. Hatzichristou D, Salpiggidis G, Hatzimouratidis K, Apostolidis A, Tzortzis V, Bekos A, et al. Management strategy for arterial priapism: Therapeutic dilemmas. J Urol. 2002;168(5):2074-2077.

16. Moscovici J, Barret E, Galinier P, Liard A, Juricic M, Mitrofanoff P, Juskiewnski S.: Post-traumatic arterial priapism in the child: a study of four cases. European $\mathrm{J}$ of Pediatric Surgery 2000;10(1): 72-76.

17. Gudinchet F, Fournier D, Jichlinski P, Meyrat B. Traumatic priapism in child: Evaluation with color flow doppler sonography. J Urol. 1992;148(2 Pt 1):380-381.

18. Mizutani M, Nakano H, Sagami K, Nihira H. Treatment of postraumatic priapism by intracavernosus inyection of alpha-stimulat. Urol. Int. 1986;41(4):312-314.

19. Visvanathan K, Burrows PE, Schillinger JF, Khoury AE. Posttraumatic arterial priapism in a 7-year-old boy: successful management by percutaneous transcatheter embolization. J Urol. 1992;148(2 Pt 1):382-383.

20. Hierro Garcia D, Silveira Paez U, Sarmentero Ortiz E. Resolución de priapismo de alto flujo postraumático mediante embolización con autocoágulo. Actas Urol Esp. 1996;20(5):471-473.

21. Gomez Vegas A, Delgado Martin JA, Blazquez Izquierdo J. Priapismo de alto flujo. VI jornada internacional e actualización urológica. Madrid. Ed. Reycosa. 1995;121-124.

22. Miller SF, Chait PG, Burrows PE, Steckler RE, Khoury AE, McLorie GA, et al. Posttraumatic arterial priapism in children: management with embolization. Radiology 1995;196 (1):59-62. 
23. Crummy AB, Ishizuka, J, Madsen PO. Posttraumatic priapism successful treatment with antologus clot embolization. AJR 1979;133(2):329-330.

24. Jameson JS, Terrry TR, Bolia A, Johnstone JM. An unusual case of priapism in a child: diagnosis and treatment. Br J Urol. 1996;77(3):462-463.

25. Sancak T, Conkbayir I. Post-traumatic high-flow priapism: management by superselective transcatheter autologous clot embolization and duplex sonography-guided compression. Journal of Clinical Ultrasond. 2001;29(6):349-353.

26. Stock KW, Jacob AL, Kummer M, Zimmermann U, Steinbrich W. High-flow priapism in a child: treatment with superselective embolization. AJR 1996;166(2):290292.

27. Gimbergues P, Raynaud F, Ravel A, Perez N, Guy L, Boiteux JP, et al. Treatment using percutaneus arterial embolization of post-traumatic priapism in children. Prog Urol. 1998;8(2):258-261.

28. Tambo M, Ohta M, Murata A, Okegawa T, Horie S, Nutahara K, et al. A case of post-traumatic high flow priapisme. Review 18 refs japanese. Japanese Journal of Urology 2003;94(5):578-581.

29. Talic RF, Al-damegh SA: Post-traumatic high-flow arterial priapism. The need for increased awareness among health care professionals. Saudi Medical Journal 2000;21(4):382385.

30. Suzuki Y, Kondo Y, Hasimoto Y, Matuzawa I, Senga Y, Akimoto M, et al. Delayed posttraumatic high flow priapism: a case report. Acta Urologica Japonica 2000;46(4): 279-281.

31. Volkmer BG, Nesslauer T, Kraemer SC, Goerich J, Basche S, Gottfried HW. Prepubertal high flow priapism: incidence, diagnosis and treatment. J Urol. 2001;166(3):10181023.

32. Gorich J, Ermis C, Kramer SC, Fleiter T, Wisianowsky C, Baseche S, Gottfried HW, Volkmer BG: Interventional treatment of traumatic priapism. J Endovasc Ther. 2002;9(5): 614-617.

33. Logarakis NF, Simons ME, Hassouna M. Selective arterial embolization for post-traumatic high flow priapism. Canadian Journal of Urology 2000;7(3):1051-1054.

34. Wang CC, Chen JH, Liu SP, Hung JJ, Liang PC, Hsieh JT. Post-traumatic, high-flow priapism treated with selective cavernous artery embolization and intracevernous streptokinase irrigation: a case report. Journal of the Formosa Medical Association 2000;99(2):952-954.

35. Alvarez Gonzalez E, Pamplona M, Rodriguez A, Garcia Hidalgo E, Nuñez V, Leiva O. High-flow priapism after blunt perineal trauma. Resolution with bucrylate embolization. J Urol. 1994;151(2):426-428.
36. Callewaert D, Stockx L, Bogaert G, Beart L. Post-traumatic high-flow priapism in a 6-year-old boy: management by percutaneous placement of bilateral vascular coils. Urology 1998;52(1):134-137.

37. Gurjal S, Mac Donagh RP, Cavanagh PM. Bilateral superselective arterial microcoil embolisation in delayed posttraumatic high flow priapism. Postgrad Med J. 2001;77 (905):193-194.

38. Brock G, Breza J, Lue TF, Tanagho EA. High-flow priapism: a spectrum of disease. J Urol. 1993;150(3):968-971.

39. Rico Lopez J, Amaya Gutierrez J, Blasco Hernandez P, Camacho Martinez E, Solaya Murillo R, Mayoral Deya A, Garcia Perez M. Priapismo de alto flujo postraumático en edad puberal. Actas Urol Esp. 1998;22(6):531-537.

40. Lee YC, Shen JT, Shin NC, Chen CC, Chou YH, Huang CH. Bilateral superselective arterial microcoil embolization in post-traumatic high-flow priapism: a case report. Kaohsiung Journal of Medical Sciences. 2003;19(2):79-83.

41. Neubauer S, Derakhshani P, Krug B, Lackner K, Heidenreich A, Engelmann V. Posttraumatic high-flow priapism in a 10-year-old boy: superselective embolization of the arteriovenous fistula. Eur Urol. 1998;33:337-339.

42. Ji MX, He NS Xang P. Use of selective embolization of the bilateral cavernous arteries for pottraumatic arterial priapism. J Urol. 1994;151:1641-1642.

43. Kim SC, Park SH, Yang SH. Treatment of posttraumatic chronic high-flow priapisms by superselective embolization of cavernous artery with autologous clot. J Trauma. 1996; 40: 462-465.

44. De Pablo Cardenas A, Jimenez Arista JI, Lozano Uruñuela F, Pinos Paul MA, Jimenez Calvo JM, Ruiz Ramo M, et al. Priapismo de alto flujo por fístula arterio-lacunar bilateral. Arch Esp Urol. 2002;55(7):821-825.

45. Millward SE, Aquino J jr, Cillimns JP. High-flow priapism recurrence after initialy successfull selective coil embolization: case report. Can Assoc Radiol J. 1997;48(2):105-107.

46. Ciampalini S, Savoca G, Buttazz L, Gattuccio J, Mucelli FP, Bertolotto M, et al. High-flow priapisme: treatment and long-term follow up. Urology 2002;59(1):110-113.

47. Ricciardi Jr, Bnatt GM, Cynamon J, Bakal CW, Melman A. Delayed high-flow priapism: pathophysiology and management. J Urol. 1993;149:119-121.

Correspondencia autor: Dr. J. Rodríguez Tolrá.

Unidad de Andrologia. Servicio de Urología. Hospital Univ. de Bellvitge. Hospitalet de. Feixa Llarga, s/n. 08907 Hospitalet de LLobregat (Barcelona). Telf: 932607600

E-mail autor: jrtol@wanadoo.es

Información artículo: Original - Disfunción eréctil

Trabajo recibido: julio 2006

Trabajo aceptado: septiembre 2006 